\title{
Oracle Database Workload Performance Measurement and Tuning Toolkit
}

\author{
Sai Peck Lee and Džemal Zildžić \\ Faculty of Computer Science and Information Technology \\ University of Malaya, Kuala Lumpur, Malaysia
}

\author{
saipeck@um.edu.my
}

\begin{abstract}
Database tuning practice is mainly conducted as a consequence of user's complaints on the performance. There is a need for a reactive monitoring and tuning tool enabling a real-time overview of the main resource consumers in order to detect and solve performance bottlenecks. With an assessment of the Oracle's high-availability database, in terms of the main architectural components and their impact on performance, we have developed a Java tool for the efficient and resource-effective tuning of Oracle databases. Our tool, called Workload Performance Monitoring and Tuning (WPMT), enables proactive and reactive database tuning. By combining today's best monitoring and tuning practices with our metrics management, we have designed a unique approach to illustrate the efficiency of the Oracle database memory segments responsible for handling the workload. This approach consists of developing database memory delta charts, illustrating the efficiency of memory initialization parameters versus component's workload performance.
\end{abstract}

Keywords: database management systems, tuning, workload performance

\section{Introduction}

Regardless of IT environment-specific requirements for running mission-critical database-driven applications, the two basic requirements are always requested: firstly, high availability, in terms of database accessibility and resource availability; and secondly, a high performance, in terms of access and code efficiency (Silberschatz, Korth \& Sudarshan, 2002). These database management systems, i.e. the most demanding systems with $24 \times 7$ availability ( 24 hours a day, 7 days a week), require regular or proactive maintenance practice in order to achieve and maintain the optimal database workload performance. Although Oracle, IBM, and Microsoft provide a solution for a high availability (or multi-node) computing environment, availability, scalability, and manageability among them differ significantly (Buch \& Cheevers, 2002; Chandrasekaran, \& Kehoe, 2003; MSDN, 2004).

Material published as part of this publication, either on-line or in print, is copyrighted by the Informing Science Institute. Permission to make digital or paper copy of part or all of these works for personal or classroom use is granted without fee provided that the copies are not made or distributed for profit or commercial advantage AND that copies 1) bear this notice in full and 2) give the full citation on the first page. It is permissible to abstract these works so long as credit is given. To copy in all other cases or to republish or to post on a server or to redistribute to lists requires specific permission and payment of a fee. Contact Publisher@InformingScience.org to request redistribution permission.
Ultimately responsible for database tuning, the most inconvenient situation for a database administrator (DBA) is when users submit complaints about the database performance. Forced to act immediately (reactive tuning), for the DBA it is a critical task due to the fact that it often happens during the peak database activity. A common solution would be to optimize the SQL (Structured Query 
Language) statement's Execution Plan that improves the execution performance. However, performing the object optimization during the peak database activity shall decrease the overall performance, since the optimization process consumes resources (CPU and RAM), and may even bring some objects temporary unavailable. Identifying the cause of performance overhead is not an easy task. For example, knowing that excessive disk I/O operations may bring down the database performance up to 2500 times (Burleson, 2003), a DBA must have a real-time overview of major I/O users, as well as the most expensive user's SQL statements causing the slowdown. The root cause can be inaccurate SQL's Execution Plan, or unbalanced memory allocation due to inappropriate initialization of database, or because the database server has no more resources to handle the workload and requires the upgrade. In any case, the DBA must be able to assess the current database resource consumption and to proceed with investigation to discover the root of the problem and to solve the problem.

Based on conducted on-line survey and an interview with Oracle Support Manager (Zildžić, 2005), we may conclude that database memory and index tuning practice needs an improvement. Since it is mainly conducted as a consequence of database performance overhead, i.e. when the user submits a complaint, a proactive monitoring of database performance should be implemented to anticipate performance leakage and ultimately avoid severe performance degradation.

The above considerations led to a custom developed monitoring and tuning tool which delivers a unique solution for the efficient and resource-effective Oracle database workload performance measurement and tuning. This tool, called Workload Performance Monitoring and Tuning (WPMT), is developed in Java. It can proactively and reactively be used during Oracle database tuning in order to measure the current workload performance of the main database components, anticipate resource shortage, and optimize database objects for the achievement of the optimal database performance (Zildžić, 2005).

\section{Oracle's Monitoring and Tuning Tools}

Effective data collection and analysis is essential for identifying and correcting system performance problems. Oracle provides tools, such as Statspack and Oracle Enterprise Manager (OEM) packs allowing a DBA to gather information on database performance (Metalink A87503-02, n.d.). These tuning tools, recommended by Oracle corporation and currently the most often used, use data dictionary views to capture database activity metrics. In this section, a part from Oracle Company designed tools, we shall assess the third-party tuning tool, i.e. the MGA's EagleEye, developed by a recognized Oracle database expert Mark Gurry.

\section{Statspack Utility}

Statspack utility consists of a set of Programming Language/Structured Query Language (PL/SQL) scripts, executed against the database, in order to gather, store data and metrics, and generates reports of the database activity. These metrics (Table 1), collected from dynamic performance tables, are automatically recorded in utility-created tables within a database with the DBA's choice of collection and threshold level to be used (Metalink 228913.1, n.d.). Bundled with the Oracle Server, it is also known as Utlbstat/Utlestat and as of database release version 8.1.6, it is renamed as Statspack. 
Table 1: Statspack utility collection levels

\begin{tabular}{|l|l|}
\hline Collection level* & Description \\
\hline 0 to 4 & $\begin{array}{l}\text { General performance metrics on all memory areas, latches, pools, and } \\
\text { events. }\end{array}$ \\
\hline 5 to 9 & $\begin{array}{l}\text { Same metrics from the lower levels, plus the most resource-intensive } \\
\text { SQL statements. }\end{array}$ \\
\hline $10+$ & Same metrics from the lower levels, plus parent/child latch data. \\
\hline
\end{tabular}

*By default and recommended value of the collection level is 5, but it depends on the scope of the tuning.

Statspack tool has been designed by the Oracle Server architects, and it generates a set of reports based on user-defined collection level. With a higher collection level, metrics capturing consisting of almost all database performance activity related segments. Executing this utility has high costs in terms of space usage and query performance. For example, just for STATS\$SYSSTAT table, Oracle will generate 255 rows for each snapshot, and there are 35 tables more. Whether required or not, the thousands of rows will be generated anyway with this utility. Managing historical metrics (dozen of snapshots) requires significant space allocation. The reports are generated in text format only, and it is difficult to establish "chain" conclusion-analysis with dependent parameters. Report analysis is a time consuming task due to non self-intuitive and complex report presentation. Statspack is not supported with database releases earlier than 8.1.6, and storing data from multiple databases in one PERFSTAT user account is currently not supported.

\section{Oracle Enterprise Manager (OEM) tools}

Oracle Enterprise Manager (OEM) is Oracle's single, integrated solution for managing, administering and monitoring global enterprises. OEM management framework consisting of three tiers: The Console (client tier) and its integrated tools provide a graphical interface for administrators to manage the complete Oracle environment. Management Servers and a database repository provide a scalable middle tier for processing system management tasks. Intelligent Agents are installed on each network node (database) to monitor the services that node provides, and to execute tasks from the Management Server (Metalink A96674-01, n.d.).

Oracle offers several tools to administer the complete Oracle environment, including: databases, Internet Application Servers (iAS), applications, and services. For this research we have assessed two types of tools that help the DBA to tune and monitor Oracle database: Performance Tuning pack (Metalink A86647-01. n.d.) with Oracle Expert tool for automated database tuning, and Diagnostics pack (Metalink A88748-02, n.d.) with a tool called Performance Manager that provides a set of graphical charts to monitor the performance of the database.

Oracle Expert provides a methodology that is used to collect, evaluate, verify, and implement database tuning changes (Metalink 93490.1, n.d.). Tuning areas cover instance parameter tuning, database structure and placement, index tuning, and SQL statement reuse evaluation. During the tuning session, Oracle Expert collects metrics from dynamic performance views, processes this input through the various rules and algorithms, and creates recommendations, tuning scripts, and reports.

Before creating a tuning session, Oracle establishes a current workload baseline (application SQL data and statistics or SQL History) of the database environment. Tuning session consists of five steps: defining the scope (SGA tuning, SQL reuse, storage management, data access - I/O), metrics collection, metrics review, recommendations based on built-in rules, and generating the SQL scripts based on the recommendations. 
Observation. Oracle Expert tool, with 900 tuning rules built-in, sub-applications SQL Analyze and Index Wizard, represents Oracle's most enhanced graphical tool, especially with the release 9i. To host and manage collected metrics, this tool creates near 700 objects: 249 tables, 270 indexes, and 63 views. Algorithms for processing recommendations based on the collected metrics are high memory and CPU consumers. Using this tool with a repository installed on a production (main) database would not be an advantage due to its negative impact on the overall database performance.

Performance Manager, available as a part of Oracle Diagnostic pack, Performance Management and Overview, provides a set of charts on the current state of the database. This tool is designed to give an immediate feedback on the state of all tuning areas. With an Overview option selected, this tool displays several charts simultaneously. Dynamic monitoring of the database state is enhanced with user defined snapshot time interval, and various type of chart formats (Metalink 169551.1, n.d.).

Observation. This tool provides a complete overview of Oracle database state, in the form of multiple-choice charts in HTML format. Also it can monitor Microsoft SQL server database. It is available for UNIX, and Windows platforms only. For the database release prior to 9i, tool is available only with OMS repository connection. With 9i release, without OMS only the current activity can be monitored. Collection and review of historical data is available with OMS repository connection exclusively. With a frequent refresh rate (snapshot time interval), the database has drawbacks in the overall performance due to increased activities (CPU and RAM consumption) necessary to calculate all performance metrics.

\section{MGA's EagleEye}

MGA EagleEye is a third-party software tool developed by Mark Gurry \& Associates (MGA) that monitors the performance of Oracle databases and provides real-time diagnostic information to allow for immediate rectification of performance problems (http://maxdbt.com/eagleeye.htm) (Gurry, 2001; Gurry \& Corrigan, 1996). This tuning tool is reviewed by 'SELECT' magazine, Australian Oracle Users Group, and recommended for users experiencing extensive problems with performance and robustness on their mission critical database.

Observation. The tool runs a set of SQL statements and records tuning information when expected response times are exceeded, including details of logged on users, the applications and specific actions they are running, and where the contention is occurring. It also produces graphical information on:

- Shared Pool Problems

- Analysis (and not rebuilt) of tables and indexes

- Waits in tables and indexes

- Sort efficiency

- Disk I/O analysis

Eagle Eye is a product that has been developed to help DBAs to quickly identify where the performance problems are occurring and the cause of the problems. When used with the Mark Gurry's books Oracle Performance Tuning and Oracle SQL Tuning, the tool enables the DBA to get performance problems under control. This tool does not provide a solution for recording historical metrics. It is designed to give an instant overview of database performance, and therefore is not suitable for a long-term proactive database maintenance. Also, it does not enable object optimization nor cache hit-ratio analysis. 


\section{Workload Performance Measurement and Tuning}

Workload Performance Measurement and Tuning (WPMT) is a custom developed monitoring and tuning tool enabling a DBA to perform reactive and proactive Oracle database tuning, with an efficient and resource-effective solution for managing the memory related historical resource and performance metrics. With an assessment of the high-availability Oracle database architectural components and their impact on workload performance, we have designed a tool with following features:

- Database connections assessment. A feature required for the successful implementation of developed tuning strategy for administrating efficiently mission-critical Oracle database-driven applications.

- Database workload assessment. Capturing and displaying in real-time the database users with the most demanding resource allocations, in terms of top memory and CPU consumers, as well as the most expensive SQL statements in terms of I/O executions.

- Instance memory allocation assessment. In order to determine the efficiency of allocated memory to manage the workload, the current memory allocation breakdown, calculated cache hit ratios using a ratio-methodology, memory sort ratio, and a redo log space requests, are calculated and displayed in real-time as well.

- Bottleneck or Wait Event assessment. With wait-methodology, we have determined total instance session's wait time and corresponding event description, required to identify the root causes of session's hang-up.

- Storage or space allocation assessment. In order to anticipate space shortage and eliminate unnecessary database performance overhead or downtime, we have developed a feature to provide an overview of the currently free/used space. Furthermore, with calculated fragmentation index, the efficiency of the overall datafile free space has been determined and displayed.

- Disk or I/O activities assessment. A real-time assessment feature detecting inappropriate or unbalanced throughput of physical storage allocation has been developed in terms of highest physical reads/writes per datafile.

- RAC inter-node performance assessment. An overview of top wait events related to instance cache has been determined using RAC's dictionary views. The efficiency of interinstance intercommunication component has been assessed using average current block time metric.

- Memory metrics management. Instead of recording metrics in database tables, and as a consequence additionally increase the database workload; we have developed a solution that uses a flat file for recording and reviewing current/historical metrics. Recorded memory related performance metrics keep track of database performance achieved with historical initialization parameters, and enable a DBA to continuously measure the efficiency of allocated memory compared to the component's workload performance (delta metrics).

- Automated object optimization feature updates the database data dictionary with the current table and index statistics required by the Oracle's Cost Based Optimizer to calculate the best SQL Execution Plan. With accurate table and index statistics, a database workload performance will be improved. 
These features successfully integrated in a user-friendly Java application (WPMT tool), enable a DBA to continuously measure, resource-effectively and efficiently, the workload performance excellence of high-availability Oracle databases.

\section{WPMT Tool's Testing}

Our tool has been tested with three workload setting criteria: Low, Medium, and High, as described in Table 2. Transactions have been executed by each user independently and simultaneously against a single CPU P-IV $1.8 \mathrm{GHz} / 512 \mathrm{MB}$ of RAM, on Personal edition Oracle 9i database. These transactions, containing a mixture of SQL commands such as INSERT, UPDATE, SELECT, and DELETE, are executed via sql scripts from Oracle SQL Plus editor.

Table 2: Workload criteria

\begin{tabular}{|l|c|c|c|}
\hline & Low & Medium & High \\
\hline Users & 2 & 5 & 13 \\
\hline Transactions & 12000 & 19500 & 43000 \\
\hline Exec. Time & $1 \mathrm{~h} 00$ & $1 \mathrm{~h} 15$ & $2 \mathrm{~h} 00$ \\
\hline
\end{tabular}

A Low workload criteria is defined as two (2) users executing 12000 transactions in one hour. A Medium workload criteria is defined as five (5) users, executing 19500 transactions within an hour and fifteen minutes. A High workload criteria is defined as thirteen (13) users, executing 43 000 transactions within two (2) hours.

Hit ratios at the beginning (start) and at the end (end) of the simulation, as well as adjusted initialization parameters SHARED_POOL_AREA (SPA) and DB_CACHE_SIZE (CACHE), are illustrated in Tables 3, 4, 5 for Low, Medium, and High workload settings respectively.

\begin{tabular}{|l|c|c|}
\hline \multicolumn{3}{|c|}{ Table 3: Low Workload* } \\
\hline & start & end \\
\hline Dic. Hit & 83,7 & 95,8 \\
\hline Lib. Hit & 99,3 & 100,0 \\
\hline Buff. Hit & 76,9 & 99,5 \\
\hline SPA & $8 \mathrm{MB}$ & $12 \mathrm{MB}$ \\
\hline CACHE & $4 \mathrm{MB}$ & $4 \mathrm{MB}$ \\
\hline \multirow{2}{*}{$* 2$ users/12 000 trans./1h } \\
\hline
\end{tabular}

\begin{tabular}{|l|c|c|}
\hline \multicolumn{3}{|c|}{ Table 4: Medium Workload** } \\
\hline & start & end \\
\hline Dic. Hit & 83,6 & 95,8 \\
\hline Lib. Hit & 99,3 & 99,9 \\
\hline Buff. Hit & 78,1 & 99,4 \\
\hline SPA & $8 \mathrm{MB}$ & $16 \mathrm{MB}$ \\
\hline CACHE & $4 \mathrm{MB}$ & $8 \mathrm{MB}$ \\
\hline$* * 5$ users/19 500 trans./1h10m \\
\hline
\end{tabular}

\begin{tabular}{|l|c|c|}
\hline \multicolumn{3}{|c|}{ Table 5: High Workload*** } \\
\hline & start & end \\
\cline { 1 - 3 } Dic. Hit & 83,9 & 95,7 \\
\hline Lib. Hit & 99,3 & 99,6 \\
\hline Buff. Hit & 76,7 & 99 \\
\hline SPA & $8 \mathrm{MB}$ & $20 \mathrm{MB}$ \\
\hline CACHE & $4 \mathrm{MB}$ & $8 \mathrm{MB}$ \\
\hline$* * * 13$ users/43000 trans./2h \\
\hline
\end{tabular}

Low Workload results, as illustrated in Table 3, show that for the achievement of an optimal database performance in case of dictionary hit $(95.8 \%)$ and a peak performance in case of library hit $(100 \%)$, a size of shared pool area initialization parameter (SPA) had to be increased from 8 to 12 MB. Furthermore, there was no need to increase a buffer cache parameter size (CACHE) since almost a peak database performance $(99.5 \%)$ has been achieved with an initial size of 4 MB (Figure 1). 


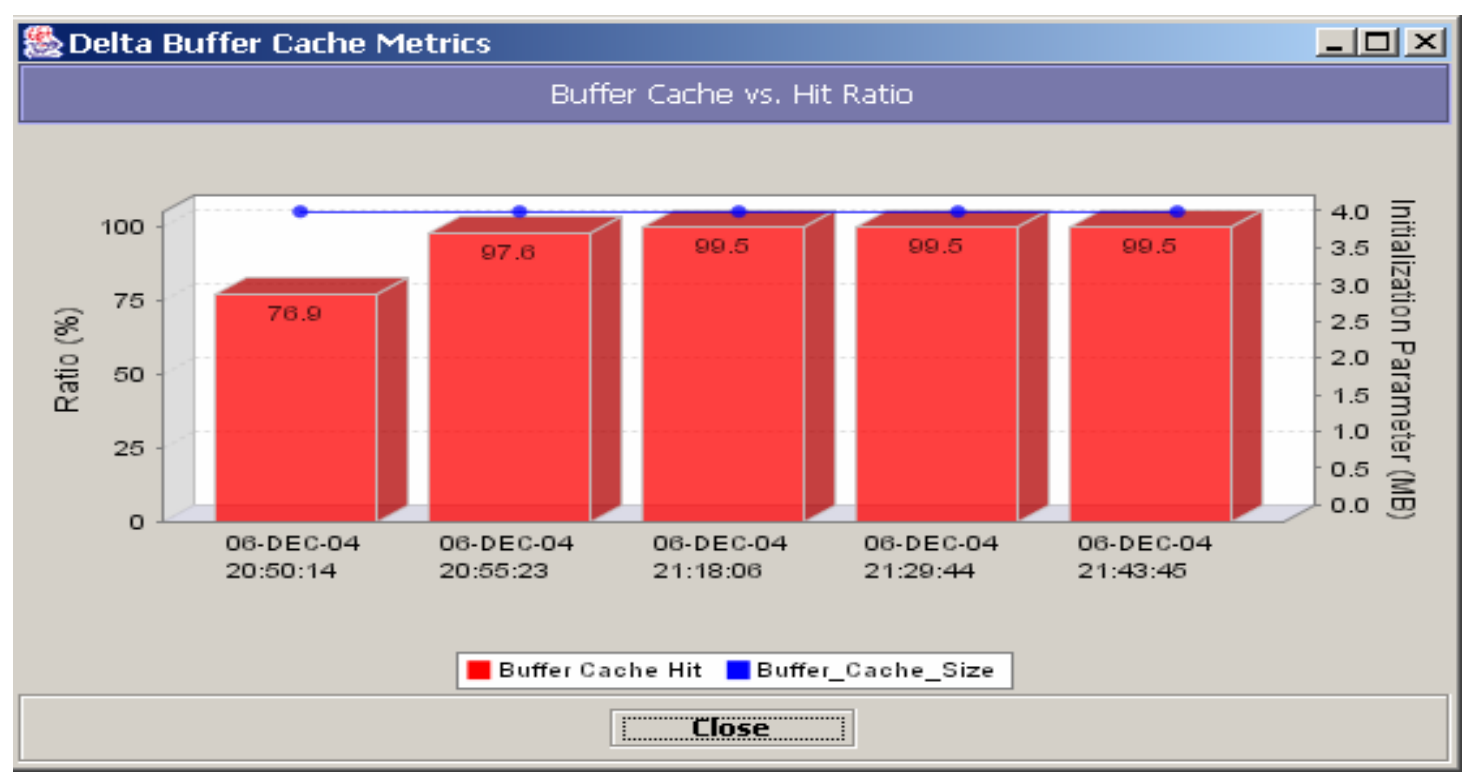

Figure 1: Buffer Hit Ratio - Low workload

Medium Workload results, as illustrated in Table 4, show that bigger values for both initialization parameters: shared pool area size (SPA) and buffer cache (CACHE), were required to achieve optimal/peak database workload performance. In case of buffer cache ratio a CACHE size was increased from 4 to $8 \mathrm{MB}$ achieving $99.4 \%$; dictionary hit ratio achieving $95.8 \%$ with $16 \mathrm{MB}$, and a peak performance for library hit ratio (99.9\%) with $16 \mathrm{MB}$ (Figure 2).

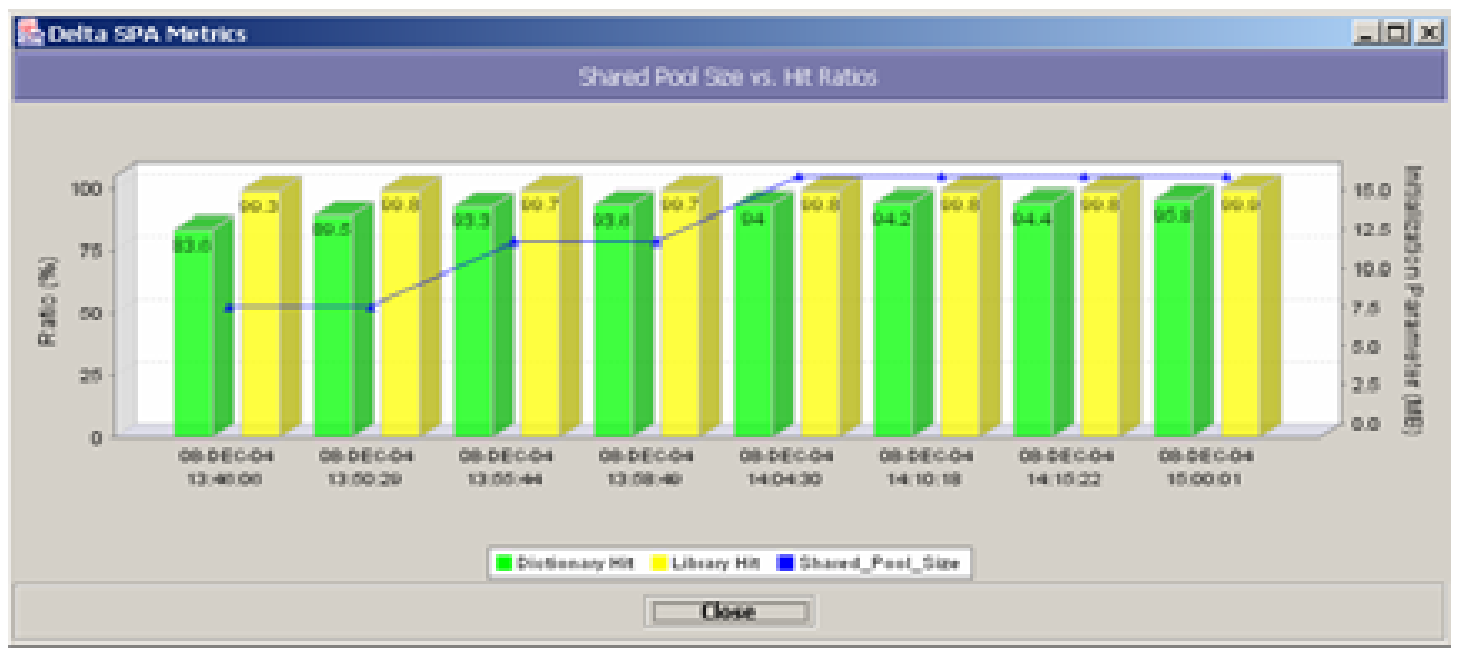

Figure 2: Library/Dictionary Hit Ratio - Medium Workload

High Workload results, as illustrated in Table 5, describe requirements, in terms of parameters size, for an achievement of optimal database workload performance. A $20 \mathrm{MB}$ of memory allocated to shared pool area (SPA) were needed to achieve $95.7 \%$ and $99.6 \%$ hit ratios for dictionary and library respectively. A buffer cache size (CACHE) was increased from 4 to $8 \mathrm{MB}$ in order to achieve $99 \%$ buffer hit ratio.

As illustrated in Tables 3,4, and 5, and as demonstrated in Figure 1 and 2, we may conclude that our WPMT tool, can be used efficiently and resource-effectively to tune database initialization parameters in order to achieve optimal/peak workload performance regardless the current database workload. 


\section{WPMT Tool's Evaluation}

Comparison between Oracle tuning tools and custom developed WPMT tool has been described in Table 6.

Table 6: Oracle database monitoring and tuning tools evaluation

\begin{tabular}{|c|c|c|c|c|c|}
\hline Features & $\begin{array}{l}\text { 1.Oracle's } \\
\text { Statspack }\end{array}$ & $\begin{array}{c}\text { 2. OEM's } \\
\text { Tuning } \\
\text { pack }(2.1)\end{array}$ & $\begin{array}{c}\text { 3. OEM's } \\
\text { Diagnostic } \\
\text { pack }\end{array}$ & $\begin{array}{l}\text { 4. MGA's } \\
\text { EagleEye }\end{array}$ & $\begin{array}{l}\text { 5. WPMT } \\
\text { v1.0 }\end{array}$ \\
\hline Workload Monitoring & $\sqrt{ }$ & $\sqrt{ }$ & $\sqrt{ }$ & $\sqrt{(4.1)}$ & $\sqrt{ }$ \\
\hline Instance Monitoring & $\sqrt{ }$ & $\sqrt{ }$ & $\sqrt{ }$ & $\sqrt{(4.2)}$ & $\sqrt{ }$ \\
\hline Bottleneck Monitoring & $\sqrt{ }$ & $\sqrt{ }$ & $\sqrt{ }$ & & $\sqrt{ }$ \\
\hline Disk I/O Monitoring & $\sqrt{ }$ & $\sqrt{ }$ & $\sqrt{ }$ & $\sqrt{ }$ & $\sqrt{ }$ \\
\hline Storage Monitoring & $\sqrt{ }$ & $\sqrt{ }$ & $\sqrt{ }$ & $\sqrt{ }$ & $\sqrt{ }$ \\
\hline RAC Monitoring & $\sqrt{ }$ & $\sqrt{ }$ & $\sqrt{ }$ & & $\sqrt{ }$ \\
\hline Object Optimization & & $\sqrt{1}$ & & & $\sqrt{1}$ \\
\hline Cumulative metrics & $\sqrt{ }$ & $\sqrt{1}$ & $\sqrt{ }$ & $\sqrt{ }$ & $\sqrt{ }$ \\
\hline Historical metrics & & $\sqrt{ }$ & $\sqrt{ }$ & & $\sqrt{(5.1)}$ \\
\hline Metrics presentation & text & recomm & graph & graph & graph/delta \\
\hline Metrics storage & database & repository & repository & & file \\
\hline Recommended tuning script & & $\sqrt{ }$ & & & \\
\hline Maintenance & reactive & proactive & proactive & reactive & $\begin{array}{l}\text { Proactive and } \\
\text { reactive }\end{array}$ \\
\hline
\end{tabular}

(2.1)Metrics are hidden and used by internal algorithms to provide recommendations in the form of tuning scripts

(4.1)Enhanced with the Explain plan feature (trace the execution of the SQL in order to discover all objects involved in the execution)

(4.2)Limited to Shared Pool Area, and no cache-hit ratio analysis

(5.1)Cache hit-ratio metrics and top initialization parameters

Oracle Statspack utility is an effective tool measuring the performance of each database component. To have an overview of metrics generated by this tool, the database must pay high costs in terms of resources, since to host the collected metrics, the utility will create additional schema in the production database. This utility maintains only the cumulative metrics, since all collected and recorded metrics are automatically deleted when the instance is restarted.

The main disadvantage of this Oracle's recommended tool for proactive maintenance, in our opinion, is an additional performance overhead created with an each utility's snapshot that collects metrics from almost all database performance views (default and recommended is level 5). For example, if we want to apply Oracle's recommended tuning methodology, i.e. one-by-one parameter, and to evaluate the effect of change on one component, Statspack's snapshot will capture metrics for other components anyway, thus increasing unnecessary database workload. Furthermore, the generated text file (report) is very complex to analyze and understand. For example, to evaluate the efficiency of hit ratios compared to dependent initialization parameters, the DBA must browse txt report from the beginning until the end since the information is provided on separate locations within a same report file. 
Oracle Enterprise Manager (OEM) tools, i.e. tuning and diagnostic packs are effective tools but should be used jointly with a repository on a remote database (separately from production database) due to high cost in resources. Using these tools independently would not be an advantage since tuning pack provides only recommendations and not an overview of performance metrics. Algorithms (rules) are hidden, and the DBA has no insight of how and why did Oracle server draw the conclusions and which parameter should be changed.

A tuning pack in combination with diagnostic pack provides a complete monitoring and tuning mechanisms to measure overall and independently database component's workload. For example, when Oracle Expert (tuning pack) recommends altering SHARED_POOL_SIZE parameters with optimal value, it will generate the script, and the DBA, after executing the script, may monitor the library and dictionary hit ratio through performance manager (diagnostic pack). Maintaining the OEM repository and tools high financial costs (new database on new server) are the main disadvantages in our opinion.

MGA's EagleEye is an efficient tool to optimize SQL statements for the best Execution Plan. The Author's book (Gurry, 2001) and EagleEye tool focus on database component that mainly measures SQL execution efficiency, i.e. Shared Pool Area. Although it is enhanced with storage and $\mathrm{I} / \mathrm{O}$ metrics, the tool is quite limited with features for measuring overall database workload performance. User-friendly GUI, low cost in resources, and a quick overview of the performance is an advantage of this tool. In our opinion, it is suitable only for proactive database maintenance.

With WPMT tool, we have combined the best practices to monitor and measure the overall database workload performance.

These best practices are the recommendations from Oracle Support Services (http://metalink.oracle.com/), combined with our 5 years expertise in maintaining the Oracle database. With a lean and portable Java user-friendly GUI, the tool has the lowest possible requirements in terms of resources. It does not create any additional table in production or remote instance, and metrics are saved in RandomAccessed flat file on the local or remote machine. Furthermore, with the most important instance related initialization parameters, recorded together with related performance metrics, we enabled historical overview of delta metrics.

\section{Summary and Conclusion}

A custom developed Java application (WPMT v1.0) combines the best practices of the most common Oracle tuning tools. WPMT's user-friendly GUI enables a DBA to efficiently perform proactive and reactive database tuning regardless of the current database workload.

Our tool combines two approaches for gauging database workload performance hit-ratio and wait (or bottleneck) analysis. Measuring a workload performance with wait analysis enables the DBA to have an insight where a database is spending time, and with hit-ratio, the efficiency of instance memory allocation is instantly detected.

Overcoming the limitations of measuring only the current (cumulative) metrics is achieved with a cost-effective and efficient solution, i.e. the flat-file metrics management. With this solution the burden of performance overhead created with a historical metrics is completely removed from a production database. Measurement with delta metrics and monitoring of memory allocation for each SGA component enables the DBA to efficiently readjust the memory for optimal database workload performance. Effective combination of the memory allocation with initialization parameter and overall efficiency of the related component responsible to manage the current workload is an advantage compared to all Oracle tools. In case of reported performance overhead (for example, low hit-ratios), based on available SGA memory, a DBA can reallocate additional memory to area(s) with low performance (SPA or Buffer Cache). 


\section{Acknowledgements}

We thank Mr. Bazran Bin Md Bahe, Oracle Support Manager Malaysia, for providing the information regarding Oracle database implementation and maintenance.

\section{References}

Buch, V. \& Cheevers, S. (2002). Database architecture: Federated vs. clustered. (Oracle white paper.) Online library: http://otn.oracle.com

Burleson, K. D. (2003). Creating a self-tuning Oracle database. Kittrell, North Carolina, USA: Rampant TechPress.

Chandrasekaran, S. \& Kehoe, B. (2003). Technical comparison of Oracle real application clusters vs. IBM DB2 UDB ESE. (Oracle white paper.) Online library: http://otn.oracle.com

Gurry, M. (2001). Oracle SQL tuning. O'Reilly \& Associates.

Gurry, M. \& Corrigan, P. (1996). Oracle performance tuning, Database management systems (2nd ed.). O'Reilly \& Associates.

Metalink A87503-02. (n.d.).Oracle9i database performance guide and reference, release 1 (9.0.1). Online: http://download-west.oracle.com/docs/cd /A91202 01/ 901_doc/server.901/a87503/toc.htm

Metalink 228913.1. (n.d.). Systemwide tuning using StatsPack reports. Oracle Support Services. On-line library: www.metalink.oracle.com

Metalink A96674-01. (n.d.). Oracle enterprise manager concepts guide release 9.2.0. On-line: http://download-west.oracle.com/docs/html/A96674_01/toc.htm

Metalink A86647-01. (n.d.). Oracle enterprise manager database tuning with the Oracle tuning pack release 9.0.1. On-line library: $\underline{\mathrm{http}} / / /$ downloadwest.oracle.com/docs/cd/A91202_01/901_doc/em.901/a86647/tun_ovw.htm\#1001025

Metalink A88748-02. (n.d.). Oracle enterprise manager getting started with the Oracle diagnostics pack release 9.0.1. On-line: http://downloadwest.oracle.com/docs/cd/A91202_01/901_doc/em.901/a88748/toc.htm

Metalink 93490.1. (n.d.). How to use Oracle Expert. Oracle Support Services. On-line library: www.metalink.oracle.com

Metalink 169551.1. (n.d.). FAQ Oracle diagnostics pack 9.0.1. Oracle Support Services. On-line library: www.metalink.oracle.com

MSDN. (2004). Federated SQL Server 2000 Servers, MSDN. Microsoft library. On-line: http://msdn.microsoft.com/library/default.asp?url=/library/en-us/architec/8 ar_cs_4fw3.asp

Silberschatz, A., Korth, H. F. \& Sudarshan S. (2002). Database system concepts (4th ed.). McGraw - Hill.

Zildžić, Dž. (2005). Workload performance measurement and tuning of high-availability Oracle databases. Masters Dissertation. University Malaya, Malaysia. 


\section{Biographies}

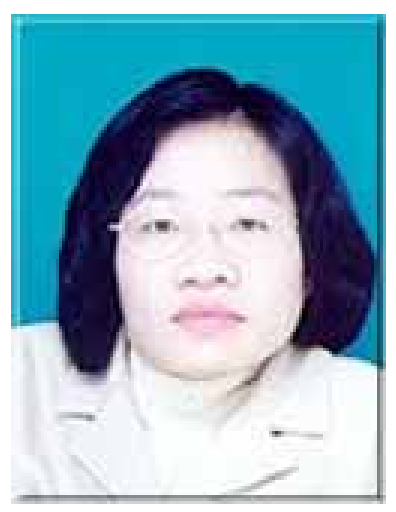

Sai Peck Lee is currently an associate professor at Faculty of Computer Science \& Information Technology, University of Malaya. She obtained her Master of Computer Science from University of Malaya, her Diplôme d'Études Approfondies (D. E. A.) in Computer Science from University of Pierre et Marie Curie (Paris VI) and her Ph.D. degree in Computer Science from University of Panthéon-Sorbonne (Paris I). Her current research interests include Software Engineering, Object-Oriented (OO) Methodology, Software Reuse and Frameworkbased Development, Information Systems and Database Engineering, OO Analysis and Design for E-Commerce Applications and Auction Protocols. She has published an academic book and more than 70 papers in various local and international conferences and journals. She had also served as the executive editor of a journal for 2 years, and has been in the programme and reviewer committees of several local and international conferences.

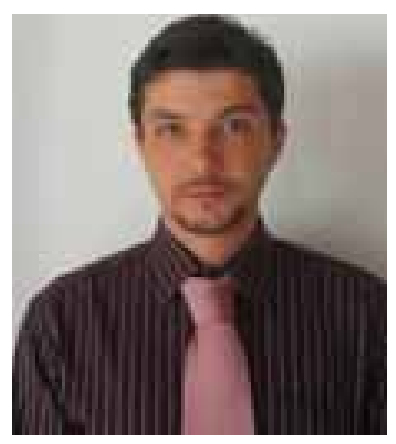

M.Sci. Dzemal Zildzic, born on 26.08.1975, Sarajevo, Bosnia and Herzegovina. Diplome Universitaire de Technology (DUT)-1997, Paris XI, France; Master of Software Engineering (MSE)-2005, Faculty of Computer Science and Information Technology (FCSIT), University Malaya, Kuala Lumpur, Malaysia. Working experience consists of OOT/Java programming and Oracle database administration (Oracle division manager - digiNET d.o.o. 2000-2002), consultant (Datapower Sdn Bhd 2004), and managerial positions (Head of IT department Bosnia Bank International 2002-2003). Since April 2005, employed as managing director of IN2 d.o.o. Sarajevo - one of the largest software development companies in the region of South-Eastern Europe. 